\title{
Telematic Requirements for a Mobile and Wireless Healthcare System derived from Enterprise Models
}

\author{
I. Widya, A. van Halteren, V. Jones, R. Bults, \\ D. Konstantas \\ Centre for Telematics and Information Technology, \\ Dept. of Computer Science, University of Twente, \\ the Netherlands; \\ widya@cs.utwente.nl
}

\author{
P. Vierhout \\ Medisch Spectrum Twente \& \\ University of Twente, \\ Dept. of Technology and Management, \\ the Netherlands
}

\author{
J. Peuscher \\ Twente Medical Systems International, \\ the Netherlands
}

\begin{abstract}
A challenge of current innovation in healthcare processes is to improve the time to treatment. This paper addresses the benefits of telematic services and mobile $\&$ wireless devices such as vital sign sensors and head mounted cameras for healthcare processes. It explores the telematic requirements for a distributed healthcare environment of Body Area Networks of the mobile devices which are connected by wireless (public) networks (e.g. GPRS and UMTS) to healthcare centres. Our main contribution is the development of Enterprise models, which are expressed in terms of the Unified Modelling Language, for the purpose of identification and justification of the telematic system requirements. The developed Enterprise models separate organizational modelling concerns such as the separation of medical roles from the agents acting in these roles. This separation enables roles to be dispatched virtually to a point of care without transporting along with all the agents, but it requires support of a telematic system to bridge the distance gap. We validate the proposed models against a trauma team scenario and consequently obtain the telematic system requirements which enable improved time to treatment for emergency services.
\end{abstract}

Keywords - ODP enterprise modelling, UML healthcare models, body area networks, wireless telematic services.

\section{INTRODUCTION}

It is well known that appropriate medical intervention during the golden-window immediately following an accident significantly improves the chance of recovery for the patient [1]. The emergency services have to get the condition of a patient under control as rapidly as possible. One of the major challenges in healthcare in general and in trauma care in particular is how to bring ICT (Information and Communication Technology) to bear in order to give added value to patient management.

This paper addresses the telematic requirements for mobile and wireless BANs (Body Area Networks incorporating devices such as vital sign sensors, microphones and cameras [2]) to improve distributed healthcare processes. An Enterprise

This work has been sponsored by the EC under the IST programme in project MobiHealth IST-2001-36006. model of healthcare processes and its refinements are used to identify where to apply ICT and to derive the aforementioned requirements.

A number of field trials and research efforts to improve healthcare processes (e.g. emergency services) address the benefits of mobile and wireless infrastructures to reduce elapsed time between symptom onset and treatment, e.g. using a cellular phone (GSM) based system [1, 3, 4]. Others apply enterprise or information models to address guidelines for healthcare process reengineering, typically emphasizing on the information system aspects and inter healthcare task relations $[5,6]$ and also [7].

In this work, we apply a primarily top-down approach using the notion of viewpoints introduced in the ODP (Open Distributed Processing) framework [8] to derive the telematic requirements. We start with an Enterprise model of healthcare processes and refine this model towards an ODP Information model. We use the refined model to directly derive the requirements for the ODP Computational and Engineering viewpoints. We therefore avoid the development of a complete ODP Information model, because elaboration of a full-blown information system is not in the scope of our work.

In the primarily top-down derivation of the requirements, we incorporate the application domain needs and we also consider the constraints of the underlying device resources and the wireless network technology. As described in [9], the underlying infrastructure typically has limited resources, e.g. bandwidth, computation power and memory. Moreover, the healthcare devices (e.g. BANs incorporating vital sign sensors) may not always be switched on, therefore they can be unknown or not available from a distributed point of view when they are powered off.

As in [5], the Enterprise models of healthcare processes described in this paper are specified in terms of healthcare tasks, roles and agents and are expressed in the Unified Modelling Language (UML) [10]. However, we capture the invariant aspects of these processes in compositional constructs to achieve correctness by construction where possible [11]. We also separate modelling concerns and distinguish between 
modelled entities that belong to the functionality of healthcare processes such as tasks and roles (which are responsible for the tasks) and the real world physical entities such as the agents assigned to the roles. In emergency services, for example, the separation of roles from agents enable roles to be dispatched virtually to the points of care without transporting all the agents, who therefore may stay at a healthcare centre. Distributed agent to agent collaborations are necessary for these mixed reality environment [12] and the availability of telematic services to bridge the distance between the points of care and the heal thcare centres are essential.

The distributed healthcare processes in general or the emergency services in particular, may therefore benefit from a kind of a low-end augmented reality environment [13, 14], which combines real world reality with virtual objects. In our case, the virtual objects are processed and communicated from a remote real world reality. For example, the real world reality may be an agent (e.g. a surgeon) at a healthcare centre viewing virtual objects, e.g. a patient's video clip and a processed heart activity diagram (ECG). The 'remote real world reality' in this case refers to the scene of the accident and for example the 12 lead ECG signals measured at several places at patient's body. This augmented reality enhances the agent's perception of the patient's condition at the remote point of care, which cannot be directly detected by the agent's senses, and helps the agent to perform his assigned role's task. However, in this paper, we do not address merging of virtual objects with real world representations as is usually done in augmented reality environments nor strive to give agents the illusion of being present at the remote points of care and able to directly and remotely perform their task (i.e. tele-presence). Rather, we focus on enabling improvement of time to treatment using telematic support to give added value, i.e. by retaining established ways of working as much as possible, in environments containing (outdoor) mobile BANs which incorporate devices with limited processing resources and limited (wireless) communication facilities.

The separation of roles from agents also implies the separation of the modelling of the patient's vital sign data from the modelling of the measurement devices. This separation is in accordance with the approach used in healthcare standardisation, for example in vital sign information representation VITAL [9], which distinguishes between devices and virtual devices. This also detaches concerns of the augmented reality environment e.g. at a healthcare centre at medical task level and the on-site real world reality at data acquisition level.

Further, we validate our Enterprise models by applying it on the MobiHealth trauma team scenario [15] to derive the Computational and Engineering requirements. We also discuss some Quality of Service aspects, which typically depend on the context of use, for example the role, the context and purpose of the task of the investigated scenario $[16,17]$.

The remainder of this paper is organized as follows. The next section describes the healthcare system and the scenario which will be used as an application context for the telematic services derived from the Enterprise models. Section 3 elaborates the Enterprise models and Section 4 discusses the requirements derived from these models. Section 5 contains the conclusions of this paper.

\section{APPLICATION}

This section briefly describes the trauma team scenario of the European project MobiHealth [15] and its communication infrastructure. MobiHealth aims in the integration of existing and forthcoming technologies like GPRS and UMTS for the development and trial of new mobile value-added healthcare services.

\section{A. Trauma Team Scenario}

If a road accident happens, a regional ambulance post dispatches an ambulance to the scene of the accident with paramedics on board. After a first assessment of the situation, the paramedics may request assistance from a mobile trauma team at a HealthCare Centre and when considered necessary members of this team (e.g. an anaesthetist or a traumatologist) may travel to the scene in a second ambulance. In this scenario, we identify three sets of actors:

- the patient,

- the paramedics, and

- the members of the trauma team (at the HealthCare Centre and in the second ambulance).

The communication and coordination between these actors are shown in Fig. 1. Bi-directional audio-visual conversation facilities will be needed between the trauma team members and the paramedic or patient at the point of care. Moreover. upstream data transfer facilities will also be needed to convey vital signs of patients to the HealthCare Centre. On the other hand, downstream messaging services are required for command and control of the multimedia devices of paramedic headset, for example to move or zoom a paramedic's headset camera towards the patient or the scene of the accident to relieve the paramedics from burdening activities.

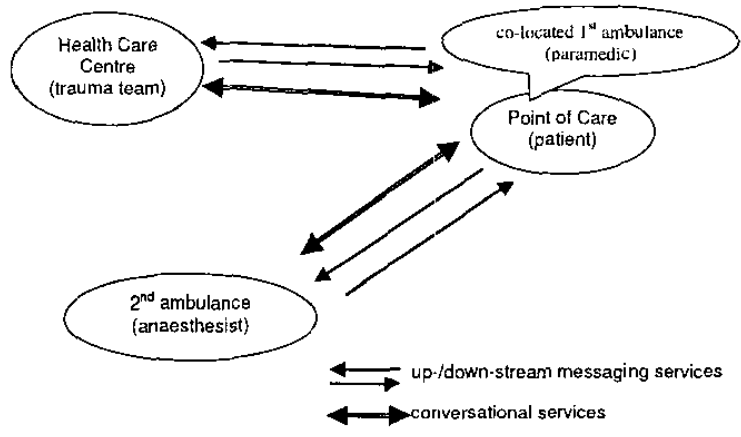

Figure $\mathrm{t}$ Interactions in Traurna Team scenario

\section{B. MobiHealth Infrastructure}

Fig. 2 shows the high-level Computational or Engineering components of the MobiHealth system. The MBUs (Mobile 
Base Units) are the gateways between patients' BAN vital sign sensing devices or the audio-video headset devices of the paramedic BAN and a secure communication platform operating on top of the (public) wired \& wireless infrastructure. The Surrogate Server intermediates as a secure high-level gateway between the HealthCare Centre node and the BANs, and among others bridges the performance hicks of the wireless communication services, as surrogate objects representing BANs run on this server.

In this setting, we observe the disjoint realities at the HealthCare Centre and at the point of care. Mobile and combined wired \& wireless telematic services bind the two realities onto a low-end augmented reality [13], enabling availability of processed vital signs measured at the point of care at the HealthCare Centre.

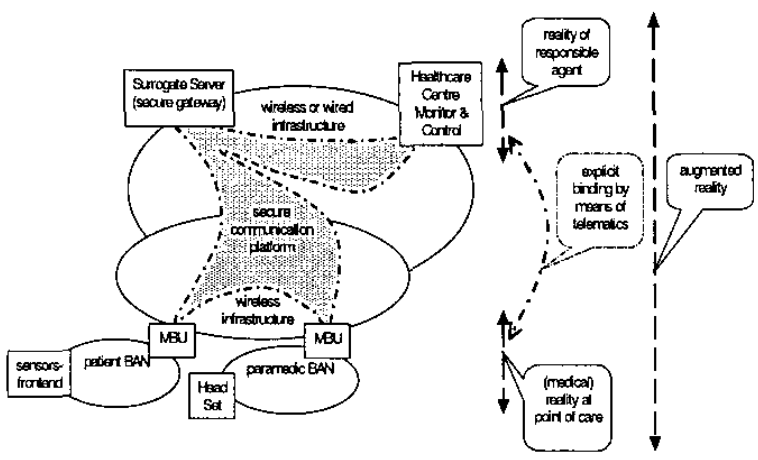

Figure 2 Mobile and wired \& wireless environment of the trauma case

\section{ENTERPRISE MODEL}

This section discusses the Enterprise model for healthcare processes step by step. We analyse current healthcare processes to align with established ways of working and to capture the basic invariant structure of medical tasks. We also investigate the relations of these tasks to the medical agents who participate in teams to provide care to patients. On the one hand, we discuss the generalization of the basic structures identified to encompass the other specialties and the inter-team relations. On the other hand, we further refine the Enterprise model by including entities related to the BANs, which incorporate mobile medical or multimedia communication devices, and we use the refinement to derive the Computational and Engineering level requirements.

\section{A. Task structure}

A healthcare process is a workflow of objective-driven tasks aimed to improve or to restore a patient's health and, in turn, a task is an embodiment of healthcare activities [5]. The following types of medical tasks are identified in a typical healthcare scenario $[18,19]$ :

- diagnostic tasks, and

- treatment tasks.
From an Enterprise modelling perspective, a diagnostic task typically contains several subtasks, each of which represents several activities:

- acquisition of a diagnostic hypothesis: e.g. by interviewing the patient in respect of their complaints (symptoms), observing the patient for the physical pathological or etiological signs and examining the patient's medical history;

- examination of the patient as a preparation task to validate the diagnostic hypothesis or to narrow-down choices if the symptoms and signs lead to several pathological or etiological diagnostic hypotheses: e.g. by laboratory tests of blood or urine samples;

- validation of the diagnostic hypothesis: e.g. by collecting and comparing test results arising out of the previous subtask.

Different diagnostic approaches can be applied and the selected approach strongly influences the diagnostic task and the breakdown of this task. In the differential diagnosis approach, a diagnosis is labelled in terms of the pathological disease [18]. The list of diseases matching the symptoms and signs in a previous diagnostic stage has to be narrowed-down, for example using additional tests. This may eventually lead to the cause. On the other hand, in the working diagnostic approach, a diagnosis is labelled in terms of symptoms and signs. Refinement is then sought in order to determine the subsequent activities. A sequence or a partial order of diagnostic activities refines the diagnosis towards the etiological or pathological cause. Preference for a certain approach depends amongst others on the medical specialty.

If the diagnostic hypothesis is confirmed, a treatment task may be initiated. This task may also be decomposed into several subtasks, e.g. treatment planning, execution and evaluation. These (sub-)tasks are often chronologically intertwined with the diagnostic tasks, since the validity of a diagnosis may need to be monitored continuously as this validity affects the treatment and moreover patient illness may develop in other directions.

An Enterprise model which can cope with hierarchically structured tasks is needed for the modelling of diagnostic processes that apply the differential approach. Moreover, if the model also can cope with partially ordered tasks, it will also be suitable for diagnostic working approaches. The need to cope with both kinds of structuring of tasks has been identified in other domains, e.g. in task-oriented educational processes [11], see also [20]. Accordingly, we reuse those task structures to model healthcare tasks (Fig. 3). For clarity of the figures, methods and attributes of classes are not shown in the UML class diagrams.

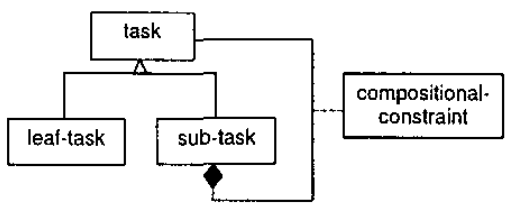

Figure 3 Healthcare Task Structure 
Fig. 3 shows that a task is either a leaf-task or a true aggregation of tasks (symbolized by the black diamond). The aggregated tasks are constrained by the compositionalconstraint, which for example represents the constraints on the permitted partial order of these tasks, on the diagnostic narrowing of the differential approach or on the decomposition of the medical objective of the encapsulating task.

The model can cope with arbitrarily deeply nested tasks, including flatly structured tasks. The hierarchical depth of a medical task structure not only depends on the complexity of the task modelled, it also depends on the task context such as the urgency of the task. The following citation illustrates the need for a flat, monolithically structured task, which contains a large set of activities in a complex medical scenario [21]:

"In dealing with the trauma victim, the physician must treat as he or she gathers information. The approach cannot be routine "take a history, do an exam, order some tests, make a diagnosis, then treat the patient." Therapeutic interventions must be made "on the fly," before the full evaluation can be completed. .......... For example, the combination of low blood pressure, unilaterally decreased breath sounds, and respiratory distress triggers a response from the physician. A chest tube is placed immediately, rather than waiting until an $\mathrm{x}$-ray can "prove" the diagnosis."

A leaf-task embodies the work-item activities associated with the care of a patient's health, for example observation, interview, preparation, treatment or validation activities mentioned earlier. An activity of a task may. require resources such as ECG graphs, blood pressure data or patient medical history (modelled by class resource and its components in Fig. 4). It may also require (logistical) facilities such as an operating theatre or an intensive care unit (class facility). From the perspective of a medical specialty, a patient is a subject which receives care of the specialty at a reference point (i.e. a particular time and location). A component of leaf-task is therefore care-receiver, which has to be assigned to a patient by the association class patients'-schedule, which contains the reference point.

In Fig. 4, the medical history (i.e. the class history) of a patient is compositionally aggregated to the patient to emphasize their tight coupling. Within a medical activity, moreover, a part of the patients' medical history may be needed as a resource. We model this latter by the class task-history, which is a component of a leaf task and which depends on the patient's history (symbolized by the dashed arrow in Fig. 4). Further, patient's medical (media) data which has been collected by a task may be stored in the patient history (i.e. history depends on med.-media in UML terms).

The model also reveals the modelling strength of UML compositional aggregations for instance compared to association classes. For example, if a patient needs an operation but the facility to operate is not available, the treatment task cannot be instantiated in the usage phase due to the compositional aggregation of leaf tasks. On the other hand, if the association class patients' schedule is not properly implemented by corresponding data flow diagrams or medical protocols, patients scheduling will be sensitive to human assignment mistakes like authentication mismatch between a task and a scheduled patient. Therefore, the class patients' schedule needs to be dependent (in UML sense) on scheduling policies which refer to medical-protocols (e.g. provide patients with identification labels, check the labels before treatment and check patients' name) or authentication protocols (this dependence is not shown in the figure).

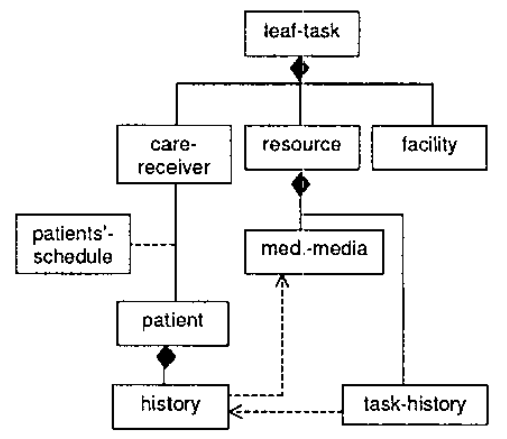

Figure 4 Leaf task structure and patient-task assignment

\section{B. Task-team structure}

As with educational tasks [11], medical tasks are organized and performed by medical agents acting in certain roles and in these roles these agents are responsible for the tasks. The class role represents a medical role (e.g. an expert in anaesthesia) and eventually has to be assigned to an agent on duty (e.g. an anaesthetist on duty). In the assigned role, this agent has the responsibility to perform a certain medical task with the authorization rights associated to the role (represented by authorization in Fig. 5).

Since complementary roles can split responsibility for a task, especially for complex and aggregated tasks, we introduce the class team either as a role or a compositionally aggregated team. The aggregation is constrained in a multi-way manner by the association class division-constraint. For example, divisionconstraint may impose how an assistant role relates to the role being assisted and specify e.g. whether an assistant anaesthetist at a point of care has the right to place a chest tube in a trauma case when the anaesthetist is located remotely at the hospital.

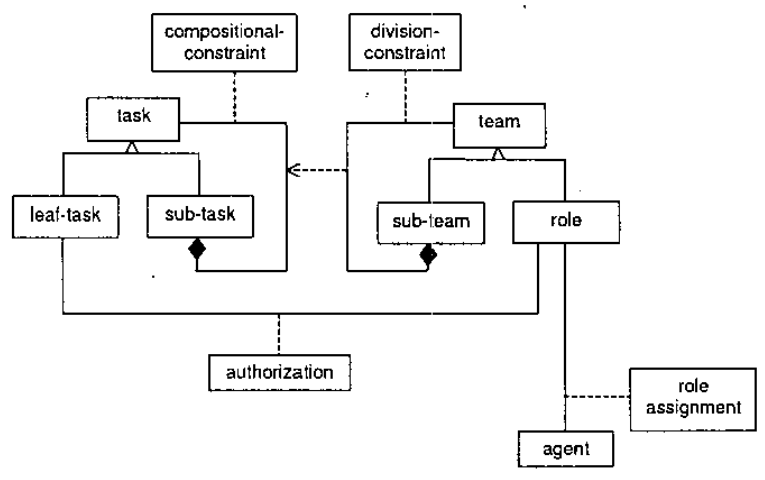

Figure 5 Task - Team structure for healthcare processes 
Furthermore, several teams may perform the same task regime concurrently, for example in road accidents involving multiple emergency teams, each of which follows the same emergency regime. Therefore, we model a team structure as a homomorphism of a task structure for scalability of the management processes (dashed arrow in Fig. 5).

\section{Team-team structure}

As mentioned earlier, the Enterprise model will be refined to bring it closer to an Information model for the purpose of deriving the requirements for the middleware support of healthcare processes directly from the refined Enterprise model.

Fig. 6 shows the team structure of Fig. 5 but extended with the association class collaboration, which specifies how teams are related to one another. Amongst others it represents the communication and coordination aspects between teams as is imposed by the association class division-constraint. The dashed anow in Fig. 6 symbolizes the dependency, which also models the breakdown of the multiparty association class division-constraint into the simpler association class collaboration. This refinement, which introduces the association class collaboration, illustrates the need for collaboration activities between agents in BAN (of sensors) based remote monitoring applications.

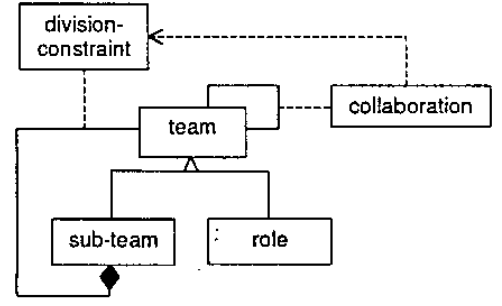

Figure 6 Inter- and intra-team collaboration

Due to the nested structure of teams, collaboration also represents the intra-team communication and coordination, e.g. referring the medical protocols that should be used between an agent at a remote point of care and an agent at the hospital as illustrated earlier by the trauma case involving the assistant anaesthetist.

\section{Task resource and BANs}

The resources of a task (e.g. ECG graphs) typically depend on the context of the task, for example, the objective of the task (e.g. validate cardiac anrest) and the role responsible for the task. Since roles are conceptual modelling entities and the agents assigned to these roles may be located remotely from the roles, assistant agents co-located with the roles have to be appointed to perform assisting activities (see Section III-C), Accordingly, we distinguish between (mental) activities related to the selection of task resources (i.e. the selection of the vital signs (e.g. ECG graphs) and their quality) and the (physical) activities related to the acquisition of the required data (i.e. the selection, attachment and the calibration of the (physical) vital sign sensing devices, e.g. the selection of a high sensitive 3 lead ECG set). The class med.-media is task resource oriented and the class medium is device oriented in Fig. 7. This separation of task-level and acquisition-level activities also provides a richer set of implementation options in a distributed environment of asymmetrical resources with respect to wireless communication and computational processing resources. This separation is also in-line with the common practice in healthcare standardization, which distinguishes between physical devices and virtual devices [9].

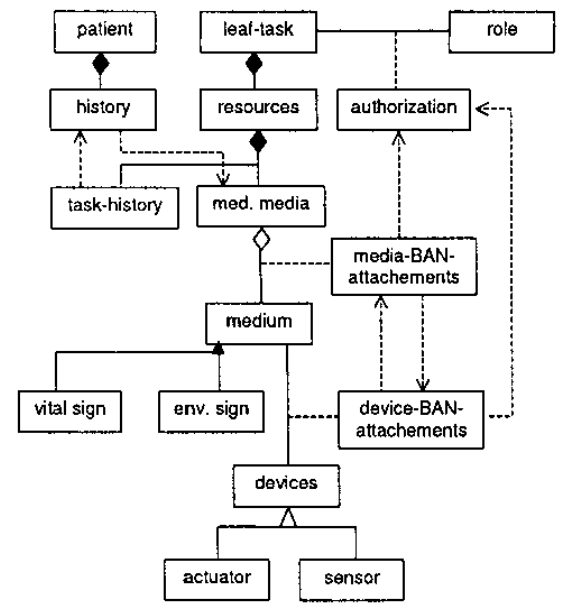

Figure 7 Task resources and the assignment classes at medical media and device levels

At task resource level, the association class media-BANattachments models the assignments of a relevant and coherent set of medical data (i.e. aggregation of medium entities, including their quality) to the resource component of a task (Fig. 7). This assignment has to be in line with the authorization rights of the role (dependence to class authorization in the figure). The class media-BAN-attachments also specify the relations between the media, for example, the priorities and the synchronization between the vital signs.

Analogously, device-BAN-attachments represent the attachment of devices (e.g. vital sign sensors) to the medical data represented by the class medium. Permissions to attach these devices also depend on the authorization rights of the role.

Both association classes are isomorphically connected to guarantee the correspondence between the devices and the vital signs. This correspondence is an elementary necessity for example to enable alignment of real and virtual entities in the augmented reality environment. Although a one-to-one association may model this isomorphism, we use two strongly connected homomorphisms to capture the dynamics of these attachments, the ripple-up of device attachments to the task resource level (e.g. in case that the request for a device or a vital sign sensor has been communicated out-of-band via an audio channel to the agent that attached the devices) and the ripple-down of medical media selections and attachments in case assignment has been initiated at task level (via a mediaBAN attachments attribute), respectively.

Agent to agent communications to realize team collaborations and remote monitoring to bridge the spatial gap 
between the location of a role and the location of the assigned agent may need BANs which incorporates multimedia devices such as cameras and microphones. The model of this BAN is shown in Fig. 8.

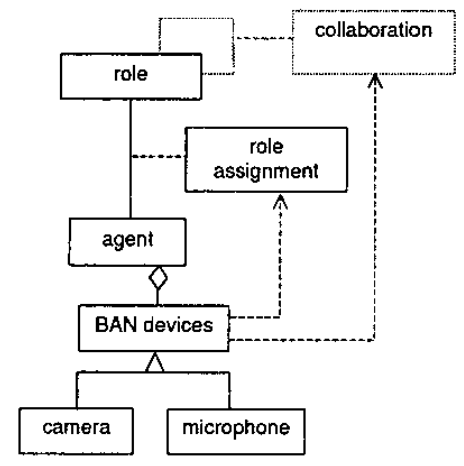

Figure 8 Paramedic BAN in a trauma case

\section{TELEMATIC REQUIREMENTS}

This section describes the telematic requirements for the trauma team scenario discussed earlier. These requirements have been derived from the (refined) Enterprise models described in the previous section, in particular from the association classes media-BAN-attachments, device-BANattachments and (team to team) collaboration. They can be categorized amongst others into the following kinds:

- Control-plane requirements: i.e. the requirements associated to the set-up of the bindings of the agent-toagent communication and the remote monitoring of vital signs that bridge the spatial gap between location of the role and the associated agent.

- Usage-plane requirements: i.e. the requirements associated to the use of the binding in respect of the communication and coordination activities in the application domain.

\section{A. Control-plane requirements}

Some of the requirements for the BAN which are associated to the set-up of the augmented reality environment by an explicit binding of the remote point of care reality towards the scope of perception or control of the agent are the following:

1) Naming \& addressing: For a UML association class to specify which instantiations of class entities are associated, the instantiations have to be uniquely identifiable and addressable in the augmented reality environment. Accordingly, a device level BAN needs to be identifiable and addressable in the (wireless) network environment. Since a healthcare BAN discussed here is centrally controlled by an MBU, which also acts as a gateway, this MBU in turn needs to be addressable in the wireless environment. Furthermore, if the devices attached to the BAN need to be addressed individually and remotely, they have to have a unique address in the particular environment. For example, if a camera head-mounted on a paramedic can be addressed and controlled remotely, the paramedic will not be burdened by requests for adjustments of the camera settings. Moreover, each ECG channel of a multilead ECG setting has to be identified individually for correct interpretation by the responsible agents. Therefore, the data acquisition front-end of the devices needs to be addressed uniquely within a centrally controlled BAN, including each of the channels of this front-end.

2) Plug-and-play (ripple up): If the MBU as a gateway is powered off, the BAN and its components are unknown in the networked environment. The MBU start-up should therefore contain a push mechanism (e.g. embodied by an announce or a registration protocol) to enable its discovery in the augmented reality environment.

Quality of Service mechanisms may be further included to enable capability alignment within the network and in-line with the application quality of service requirements.

3) Adaptable communication: In outdoor cases, communication resources are typically scarce. Amount, quality and coherence of the data to be exchanged have to match with the limitations of the communication channel capability. Set up of tailorable and adaptable telematic services is typically required in these environments to better cope with bandwidth limitations and variations in communication enors like data loss and channel dropouts. Buffers, data prioritising, synchronization mechanisms and data acknowledgements may be needed in these environments. For example, if TCP is used in the combined wired and wireless (e.g. GPRS coding schema 1) environment, the congestion control parameters of TCP also need to be aligned to the delay and delay variations of the wireless part.

\section{B. Usage-plane requirements}

The requirements for the BAN in respect of the transfer of medical data are amongst others:

1) Upstream push mechanism: Dependence of mediaBAN-attachments to device-BAN-attachments requires a push mechanism, such as a plug-and-play or a call-back mechanism, which enables the visualization (remote monitoring) of vital signs, for which an on-site medical agent has attached corresponding sensors at a remote point of care. However, the agents may also communicate some vital sign data via an audio or video channel, for example patients' facial colour as a trend vital sign for brain oxygenation (cyanosis).

Additionally, a (block-based) streaming protocol may be needed to upstream sets of coherent continuous-time vital signs or multimedia data. This also generates the need for several preservation mechanisms like (time and event) synchronization, data priority and data accuracy when using layered compression.

2) Downstream messaging mechanism: Dependence of device-BAN-attachments to media-BAN-attachments requires a messaging method that ripples down the choice for a specific task resource to the MBU to alert an on-site medical agent or a 
patient at the remote point of care to attached a corresponding sensor or to require an off-site medical agent to forward a configuration setting to a sensor or actuator device. Remote camera control described earlier also emerges from this dependence.

The requirements for the BAN in respect of the team collaboration or patient to team communication depend on the healthcare context. A GSM based channel often satisfies the requirements for communication between a paramedic and a trauma specialist in the trauma case. On the other hand, conversation between a patient and a speciakist may need better quality channels. High fidelity audio channels may help in case patients need to be calmed down or reassured and video channels may improve these conversations further.

\section{Refined trauma team scenario requirements}

The scenario described in the previous section also shows the interactivity of the communication between the remotely located agents including the transfer of the clinical patient data to the healthcare centre. This means that upstream and down stream data transfers are often dependent on one another and transfer delays therefore should not exceed the boundaries given by human factor studies (e.g. $500 \mathrm{~ms}$ for data retrieval applications and $150 \mathrm{~ms}$ for conversational applications). This constrains the audio-video communication quality in the wireless environment, it also constrains the quality of the vital signs which can be transferred. For example, an accurate 12 lead over-sampled ECG set already requires a $64 \mathrm{kbits} / \mathrm{s}$ UMTS channel (approximately, 8 time-slots of GPRS channels of Coding Schema 1) for real-time communication and, on the other hand, currently available GPRS terminals typically support 1 or 2 time-slots for upstreaming data.

Moreover, several types of vital signs for emergency services are interdependent, together they form a unit of interpretation due to the typical indirect way of measuring patient condition. For example, oxygenation of the brain is usually estimated from oxygen saturation $\left(\mathrm{O}_{2}\right.$ sat $)$ measured at a fingertip surface by beaming for blood colour, respiration rate including a $\mathrm{CO}_{2}\left(\mathrm{pCO}_{2}\right)$ measurement, temperature, blood pressure and heart rate or ECG measurements. However, as a first indication (trend sign) of oxygenation the patient's facial colour is observed for signs of cyanosis. In the estimation of brain oxygenation, validity of the measured $\mathrm{O}_{2}$ sat depends on the patient's temperature. Moreover, heart rate as a trend sign of ECG has a higher priority than ECG. So the latter may be dropped or deferred in case of communication dips. The previously mentioned vital signs are typical for emergency services.

Table 1 illustrates the vital signs and their quality of the telematic service requirements of emergency services $[1,4,6]$. As shown in the table, some vital signs can be communicated by the paramedics to the trauma team at the HealthCare Centre via audio or video channels, for example pupil reaction, facial colour and also the (Glasgow Coma) trauma score. Other important information for a remotely located trauma team is still pictures or video of the scene of the accident to give an indication of patient's condition from the damage of the vehicle and the patient's positions in the vehicle. Today, polaroid pictures are taken and are handed over on the patient's arrival at the hospital A\&E department. Wireless transfer of these types of information from the scene will enable teams and facilities to be prepared before patient's arrival, and thus improve time to treatment of the patient on arrival at the hospital.

Table 1 also shows that a $64 \mathrm{kbps}$ UMTS channel is more than sufficient to convey a typical set of vital signs for emergency services. Higher bandwidth may be needed for more accurate vital signs or in case video monitoring of patient's movement or facial expression or colour is required.

TABLE 1 EMERGENCY SERVICES VITAL SIGNS (12 BITS ACCURACY)

\begin{tabular}{|c|c|c|c|c|}
\hline vital sign & $\begin{array}{l}\text { vital sign } \\
\text { type }\end{array}$ & sensors & quality aspects & $\begin{array}{c}\text { quality of } \\
\text { service }\end{array}$ \\
\hline \multirow[t]{3}{*}{ heart } & $\begin{array}{l}\text { heart beat } \\
\text { rate }\end{array}$ & $\begin{array}{l}\text { thumb } \\
\text { sensor }\end{array}$ & $\begin{array}{l}\text { numerical; } \\
\text { continuously; } \\
\text { priority } 10 ;\end{array}$ & low bitrate \\
\hline & $\begin{array}{l}\text { heart beat } \\
\text { curve }\end{array}$ & & $\begin{array}{l}100 \mathrm{~Hz} \text {; } \\
\text { continuously; } \\
\text { priority } 10 \text { if } \\
\text { heart beat rate } \\
\text { not present; }\end{array}$ & $2400 \mathrm{bps}$ \\
\hline & $\begin{array}{l}\text { ECG heart } \\
\text { beat curve }\end{array}$ & $\begin{array}{l}3 \text { lead, } \\
2 \text { channels }\end{array}$ & $\begin{array}{l}100 \mathrm{~Hz} \\
10 \text { seconds; } \\
\text { priority } 5\end{array}$ & $\begin{array}{l}4800 \mathrm{bps} / 10 \mathrm{~s} ; \\
5 \mathrm{kB} \text { data if } \\
\text { uncompressed }\end{array}$ \\
\hline $\begin{array}{l}\text { blood } \\
\text { pressure }\end{array}$ & & & $\begin{array}{l}\text { numerical; } \\
\text { every } 5,10,30, \\
60 \text { min.; } \\
\text { prionity } 10 ;\end{array}$ & low bitrate \\
\hline temperature & & & $\begin{array}{l}\text { numerical; } \\
\text { every } 15 \text { min.; } \\
\text { priority } 7\end{array}$ & low bitrate \\
\hline $\mathrm{O}_{2}$ saturation & & & $\begin{array}{l}\text { every } 15 \text { min.; } \\
\text { priority } 10 ;\end{array}$ & 480 bps \\
\hline $\mathrm{pCO}_{2}$ & & & $\begin{array}{l}\text { numerical; } \\
\text { every } 15 \text { min.; } \\
\text { priority } 10 ;\end{array}$ & low bitrate \\
\hline $\begin{array}{l}\text { respiratory } \\
\text { rate }\end{array}$ & & & $\begin{array}{l}\text { numerical; } \\
\text { priority } 7\end{array}$ & low bitrate \\
\hline trauma score & & & $\begin{array}{l}\text { numerical; } \\
\text { once \& if } \\
\text { patient's } \\
\text { condition } \\
\text { changes; }\end{array}$ & low bitrate \\
\hline pupil reaction & \multicolumn{2}{|c|}{$\begin{array}{l}\text { audio message (or } 2 \\
\text { photos) }\end{array}$} & \multicolumn{2}{|c|}{$\begin{array}{l}\text { via paramedic BAN headset; if } \\
\text { photos, see cells below; }\end{array}$} \\
\hline facial color & oxygenation & $\begin{array}{l}\text { photo } \\
\text { camera } \\
\text { (video is } \\
\text { much } \\
\text { better) }\end{array}$ & $\begin{array}{l}\text { once \& if } \\
\text { patients' } \\
\text { condition } \\
\text { changes; } \\
\text { Resolution } 100 \\
\text { pixels/cm, true } \\
\text { colour } \\
\text { (24b/pixel); }\end{array}$ & $\begin{array}{l}2,6 \mathrm{Mb} \text {-data } \\
\text { luminance } \\
\text { compressed } \\
(9 \times 12 \mathrm{~cm}) \\
\text { or } 40 \text { seconds } \\
\text { via } 64 \mathrm{kbps} \\
\text { line }\end{array}$ \\
\hline
\end{tabular}

\section{CONCLUSIONS}

This paper discusses the telematic service requirements for a mobile and wireless environment of BANs incorporating medical and multimedia devices to support and to improve distributed healthcare processes. These requirements are derived using a primarily top-down approach in-line with the ODP viewpoints framework. 
The developed and proposed Enterprise models capture the organisational invariants of healthcare processes in UML compositional constructs in terms of roles, agents and tasks. This paper emphasises the modelling of roles and agents instead of tasks and task relations because neither healthcare process reengineering nor developing an information system for healthcare is in the scope of the work. The modelling separation of roles and agents reveals the opportunities to apply telematic services because roles could be dispatched to a point of care while some of the associated agents remained at the healthcare centre and retained their established ways of working as much as possible.

Moreover, the described telematic services bind BANs of healthcare devices at points of care to remote healthcare nodes and bring measured and captured medical and multimedia data to the scope of agents perception, therefore enabling the improvement of time to treatment in an augmented reality way.

The proposed approach has however the implication that even in a monitoring type of a medical task, the dynamics of the task activities in a distributed case require collaboration support, for example to enable coordination and communication between on-site and off-site agents (Section III-C).

The elaborated requirements of the trauma team scenario show the feasibility and added value of the mobile and wireless environment to improve emergency services. However, the derived requirements and the capabilities of the underlying mobile and wireless infrastructure seem to have mismatching characteristics. Provided (wireless) communication channels are often configured asymmetrically with a higher downstream bandwidth, but the applications require high bandwidth upstream channels. Moreover, the applications typically fetch data sets from the mobile devices, which have limited resources and are intermittently online. Internet protocols, which apply the client-server paradigm with typically a thin client and heavy server, could not be used in a straightforward manner. Current and future work therefore focuses amongst others on the use of flexible coding of vital sign data, automated lookup, discovery and administration of BANs supported by suitable Internet protocol stacks.

\section{REFERENCES}

[1] Gagliano, D.M. and Xiao, Y., "Mobile Telemedicine Testbed" in Proc 1997 American Medical Informatics association (AMIA) Annual Fall Symposium, pp.383- 387, National Library of Medicine Project N0-1LM-6-3541, 1997;

[2] Jones, V., Bults, R., Konstantas, D., Vierhout, P.A.M., "Healthcare PANs: Personal Area Networks for trauma care and home care" in Fourth International Symposium on Wireless Personal Multimedia Communications, Sep. 9-12, 2001, Aalborg, Denmark;

[3] Cullen, J. et al., "Wireless Mobile Telemedicine: En-Route Transmission With Dynamic Quality-of-Service Management", in Telemedicine and Telecommunications: Options for the New Century, 2001, March 13-14;

[4] Bisain, A.S., "Intelligent Network Based Wireless Services for Telemedicine", Master of Science report, Dep. of System Engineering, Graduate School of the University of Maryland, 1998, 73 pp.;

[5] Luzi, D., Ricci, F.L., and Serbanati, L.D., "Extending the Standard Architecture for Healthcare Units: The Guideline Server", in New
Technologies in Hospital Information Systems, J. Dudeck et al. (Eds.). IOS Press, 1997, pp. 95 - 101;

[6] Leisch, E. and Orphanoudakis, S., "HECTOR Solutions" in $I^{\text {" }}$ International Conference on Health Emergency Telematics, Sevilla, Spain, March 15-17, 1999, 17 pp.;

[7] NHS-HCMP, "Healthcare Models", NHS Heatth Care Modelling Programme home page http://www.standards.nhsia.nhs.uk/hcm/ index.html, 2000;

[8] Blair, G., and Stefani, J-B., Open Distributed Processing and Multimedia, Addison-Wesley, 1998;

[9] Norgall, T., "VITAL - towards a Family of Standards for Open Medical Device Communication" in XIII Intemational Conference Computing in Clinical Laboratories, Milan, 21-23 September, 2000;

[10] Booch, G., Rumbaugh, J., and Jacobson, I., "The Unified Modeling Language User Guide", Addison-Wesley, 2001;

[11] Widya, I., Volman, C., Pokraev, S., De Diana, I. and Michiels, E. "Enterprise Modelling for an Educational Information Infrastructure", in Enterprise Information Systems III, J. Filipe et al. (eds.), Kluwer Academic Publ., 2002, pp. 231 - 239;

[12] Azuma, R.T., "A Survey of Augmented Reality", in Presence: Teleoperators and Virtual Environments, 6(4), 1997, pp. 355 - 385;

[13] Milgram, P. and Kishino, F., "A Taxonomy of Mixed Reality Visual Displays", IEICE Transactions on Information Systems, vol. E77-D, no. 12, Dec. 1994;

[14] Sheridan, T.B., "Interaction, Imagination, and Immersion Some Research Needs", Proceedings of the ACM Symposium on Virtual Reality Software and Technology, Seoul, Korea, 2000, pp. 1 - 7;

[15] Bouch, A., and Sasse, M.A., "Network Quality of Service - An Integrated Perspective", Proc. RTAs'99, Vancouver, 1999;

[16] Hesselman, C., Widya, I., van Halteren, A., and Nieuwenhuis, L.J.M. "Middleware support for media-strearning establishment driven by useroriented QoS requirements", in Proceedings of the Interactive Distributed Multimedia Systems and Telecommunication Services, Enschede, Oct. 2000, pp. 158 - 171, Springer Verlag LNCS 1905;

[17] MobiHealth, "MobiHealth project IST-2001-36006", EC programme IST, 2002, http://www.mobihealth.org;

[18] Hobsley, M., "Pathways in Surgical Management", Edward Arnolds Pub. Lid., London, 1979;

[19] Ewos/Etg068, "Multimedia Medical Data Interchange", European Workshop for Open Systems, Brussels, 1996;

[20] Yates, M. et al., "TiNA Business Model and Reference Points - version 4.0", May 22nd, 1997, http://www.tina.com:

[21] Argyle, B., "New York Emergency Room RN: General Approach to Trauma", http://www.nyerrn.com/er//g.htm, 1996; 